\title{
Automated segmentation of hepatic vessel trees in non-contrast X-ray CT images
}

\author{
Suguru Kawajiri ${ }^{a}$, Xiangrong Zhou ${ }^{a}$, Xuejun Zhang ${ }^{a}$, Takeshi Hara ${ }^{a}$, Hiroshi Fujita ${ }^{a}$, Ryujiro \\ Yokoyama $^{b}$, Hiroshi Kondo ${ }^{b}$, Masayuki Kanematsu ${ }^{b}$ and Hiroaki Hoshi ${ }^{b}$ \\ ${ }^{a}$ Department of Intelligent Image Information, Division of Regeneration and Advanced Medical \\ Sciences, Graduate School of Medicine, Gifu University, 1-1 Yanagido, Gifu 501-1194, Japan; \\ ${ }^{b}$ Department of Radiology, Gifu University Hospital, 1-1 Yanagido, Gifu 501-1194, Japan
}

\begin{abstract}
Hepatic vessel trees are the key structures in the liver. Knowledge of the hepatic vessel trees is important for liver surgery planning and hepatic disease diagnosis such as portal hypertension. However, hepatic vessels cannot be easily distinguished from other liver tissues in non-contrast CT images. Automated segmentation of hepatic vessels in non-contrast CT images is a challenging issue. In this paper, an approach for automated segmentation of hepatic vessels trees in non-contrast X-ray CT images is proposed. Enhancement of hepatic vessels is performed using two techniques: (1) histogram transformation based on a Gaussian window function; (2) multi-scale line filtering based on eigenvalues of Hessian matrix. After the enhancement of hepatic vessels, candidate of hepatic vessels are extracted by thresholding. Small connected regions of size less than 100 voxels are considered as false-positives and are removed from the process. This approach is applied to 20 cases of non-contrast CT images. Hepatic vessel trees segmented from the contrast-enhanced CT images of the same patient are used as the ground truth in evaluating the performance of the proposed segmentation method. Results show that the proposed method can enhance and segment the hepatic vessel regions in non-contrast CT images correctly.
\end{abstract}

Keywords: Non-contrast X-ray CT images, hepatic vessels, human body segmentation, image processing

\section{INTRODUCTION}

The latest multi-slice CT scanners generate a large number (500-1000) of slices to construct a volumetric CT image covering a wide volume of human body in a very short time (10-20 seconds). Although such a volumetric CT image can provide detailed information of human internal organs, the interpretations (viewing 800-1200 slices of CT images manually in front of a monitor for each patient case) need a lot of time and energy. Therefore, computer-aided diagnosis (CAD) systems which can support the multi-lesion interpretations for multi-organs in a CT image are desirable. Such CAD system can increase the lesion detection accuracy of radiologist and decrease the interpretation burden. Anatomical human structure recognition in CT images is necessary for the development of such CAD systems.

Liver is one of the most important diagnosis target organs of CAD systems. Lesion detection and surgery planning of the liver always require CAD systems to extract the liver region first and recognize the hepatic vessel trees in CT images. Many research works ${ }^{1-6}$ were presented for liver segmentation and hepatic vessels recognition based on contrast-enhanced or multi-phase abdominal CT images. However, few of them focused on the liver segmentation and vessel recognition using non-contrast CT images that are routinely used for lesion screening in clinical medicine.

We have proposed an automated approach for liver region segmentation in non-contrast CT images using a probabilistic atlas and confirmed the efficiency and accuracy of the method based on a large database ${ }^{7-9}$. In this paper, we propose a method to extract the hepatic vessels including portal vein and hepatic vein in non-contrast CT images in the preparation of analysis of liver lobe recognitions. The intensity enhancement and Hessian based line filtering are two principal processes. In the following sections, we first describe the outline of hepatic vessels extraction and show the details of our approach in Section 2. Experimental results and discussions are presented in Section 3 and 4, respectively. A conclusion is given in Section 5.

Corresponding author information:

E-mail: zxr@fjt.info.gifu-u.ac.jp, Telephone: +81-58-230-6510

Medical Imaging 2007: Image Processing, edited by Josien P. W. Pluim, Joseph M. Reinhardt, Proc. of SPIE Vol. 6512, 65123A, (2007) · 1605-7422/07/\$18 - doi: 10.1117/12.710343 


\section{METHODS}

In non-contrast CT images, hepatic vessels have a lower CT number when compare to other liver tissues. However, the difference in CT number is very small and differentiation between hepatic vessels and other liver tissues based on computer analysis of CT numbers is difficult. The differentiation between hepatic vessels and other liver tissues in non-contrast CT images is difficult even for human observer. CT images enhanced with contrast materials are usually obtained for the visualization of blood vessels. In this paper, vessels in non-contrast CT images ware enhanced using a required Gaussian kernel function. The vessels region were then extracted using a Hessian matrix ${ }^{10,11}$.

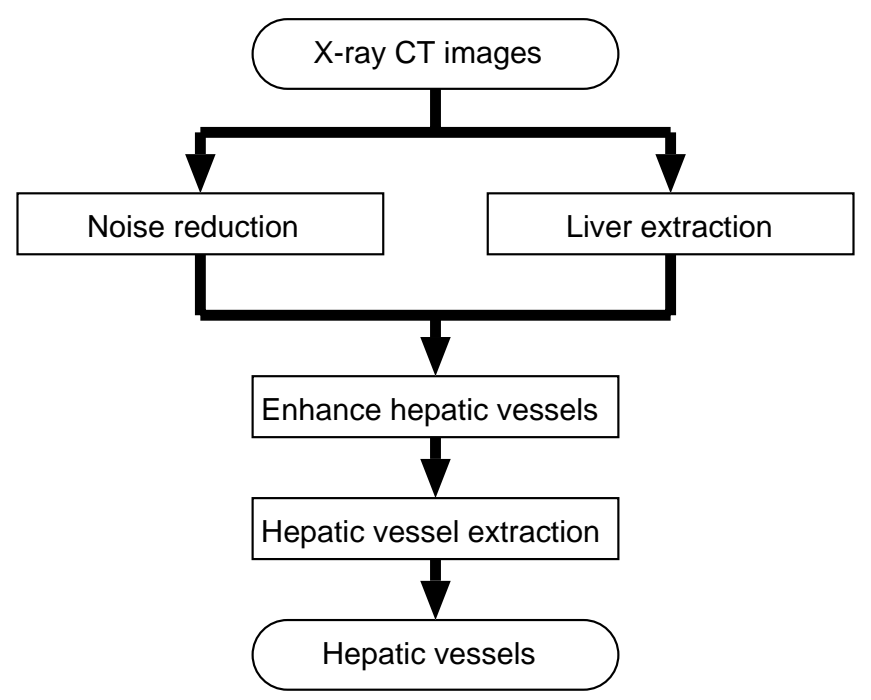

Figure 1. The flow diagram of hepatic vessel enhancement.

\subsection{Noise reduction and liver region extraction}

Noise reduction was performed using a filtering technique. A number of image filters were considered including the Gaussian filter and the Median filter. The median filter of size $7 \times 7 \times 7$ was chosen in this study because of its effectiveness in noise reduction.

Liver region areas were extracted using the method proposed by Zhou et $\mathrm{al}^{9}$. In that method, organ-of-interest were extracted using an 'Atlas'-based approach. Probabilistic distribution of gray-scale level and existence ware established using a sample of 77 cases. The probabilistic distributions were transformed and applied to individual cases in this study in extracting the liver regions.

\subsection{Enhancement of hepatic vessels}

\subsubsection{Enhancement of hepatic vessels using histogram transformation}

Let $\mu$ and $\sigma$ be the mean and standard deviation of gray-scale level of hepatic vessel, respectively. A vessel-enhanced image $p(\boldsymbol{x})$ is enhanced selectively by a transformation of image $I(\boldsymbol{x})$ using Equation (1).

$$
p(\boldsymbol{x})=C \exp \left[-\frac{\{I(\boldsymbol{x})-\mu\}^{2}}{2 \sigma^{2}}\right]
$$

where $C$ is a non-zero constant value.

Equation (1) is a windowing function of voxel value $I(\boldsymbol{x})$ for histogram transformation. The mean $\mu$ and standard deviation $\sigma$ of gray-scale level of hepatic vessels were estimated as follows: First, the whole liver region area without hepatic vessels was estimated using the method proposed by Zhou et $\mathrm{al}^{9}$. Second, a difference area was obtained by subtracting the liver region without the hepatic vessels from the whole liver area. 
As a difference area may include a lot of hepatic vessels, the mean of hepatic vessels are estimated by calculating the mean, standard deviation, and histogram of the difference area. Therefore, we assumed gray-scale level of hepatic vessel as normal distribution because difference area was nearly equal to normal distribution.

\subsubsection{Enhancement of hepatic vessels using line-filter based on Hessian matrix}

Hepatic vessel area has possibility of inclusion of non-hepatic vessels. We have assumed that hepatic vessels composite set of line or cylinder components and have tree structures, so we had discussed a method for hepatic vessel components enhancement which can extracted features of line or cylinder components from pre-enhanced area selectively. There are a lot of method which enhance various size of cylinder or line such as Tensor Voting ${ }^{12}$ or using eigenvalues of Hessian $10,11,13,14$. Finally, the method proposed by Sato et al ${ }^{11}$ was used to our method.

Let $I$ be a 3-dimensional gray-scale isotropic image and $I(x)$ be a gray-scale value at coordinate $x=(x, y, z)$.

The eigenvalues of Hessian $\nabla^{2} I(x)$ is written as,

$$
\nabla^{2} I(\boldsymbol{x})=\left[\begin{array}{ccc}
I_{x x}(\boldsymbol{x}) & I_{x y}(\boldsymbol{x}) & I_{x z}(\boldsymbol{x}) \\
I_{y x}(\boldsymbol{x}) & I_{y y}(\boldsymbol{x}) & I_{y z}(\boldsymbol{x}) \\
I_{z x}(\boldsymbol{x}) & I_{z y}(\boldsymbol{x}) & I_{z z}(\boldsymbol{x})
\end{array}\right],
$$

where $I_{x x}(\boldsymbol{x})$ represents partial derivatives of $I(\boldsymbol{x})$.

For the calculation of partial derivatives, $I(\boldsymbol{x})$ was convoluted with $G(x)=(1 / \sqrt{2 \pi} \sigma) \exp \left(-x^{2} / 2 \sigma^{2}\right)$ or its derivatives. Our method uses recursively implement of Gaussian ${ }^{15}$ in convolution process to improve computing cost.

If eigenvalues of $\nabla^{2} I(\boldsymbol{x})$ are $\lambda_{1}, \lambda_{2}, \lambda_{3}\left(\lambda_{1} \geq \lambda_{2} \geq \lambda_{3}\right)$, then

$$
S_{\text {line }}=\left\{\begin{array}{ll}
\left|\lambda_{3}\right| \psi\left(\lambda_{2} ; \lambda_{3}\right) \omega\left(\lambda_{1} ; \lambda_{2}\right), & \lambda_{3} \leq \lambda_{2}<0 \\
0, & \text { otherwise }
\end{array},\right.
$$

where

$$
\begin{gathered}
\psi\left(\lambda_{s} ; \lambda_{t}\right)= \begin{cases}\left(\frac{\lambda_{s}}{\lambda_{t}}\right)^{\gamma_{\psi}}, & \lambda_{t} \leq \lambda_{s}<0, \\
0, & \text { otherwise }\end{cases} \\
\omega\left(\lambda_{s} ; \lambda_{t}\right)= \begin{cases}\left(1+\frac{\lambda_{s}}{\left|\lambda_{t}\right|}\right)^{\gamma_{\omega}}, & \lambda_{t} \leq \lambda_{s} \leq 0 \\
\left(1-\alpha \frac{\lambda_{s}}{\left|\lambda_{t}\right|}\right)^{\gamma_{\omega}}, & \frac{\left|\lambda_{t}\right|}{\alpha}>\lambda_{s}>0 \\
0, & \text { otherwise }\end{cases}
\end{gathered}
$$

$\gamma_{\psi} \geq 0, \gamma_{\omega} \geq 0$ and $0<\alpha \leq 1.0$.

$S_{\text {line }}$ shows that similarity of line components using eigenvalues of Hessian. If the voxel is not a part of hepatic vessels, $S_{\text {line }}$ will indicate low value (ideal value is zero).

$S_{\text {line }}$ can be calculated by any value of parameter $\sigma$. Parameter $\sigma$ is corresponded to diameter of vessels which the method wants to enhance. In this method, we have chosen $\sigma_{i}$ using geometric series which have first term $\sigma_{0}$ and common ratio $s$ as follow: ${ }^{11}$

$$
\sigma_{i}=s^{i-1} \sigma_{0},
$$

Finally, filter responses convoluted Gaussian with various values of $\sigma$ are integrated into $M_{x}$,

$$
M(\boldsymbol{x})=\max _{\sigma_{i}} \sigma_{i}^{2} S_{\text {line }}\left(\boldsymbol{x} ; \sigma_{i}\right),
$$

where $S_{\text {line }}\left(\boldsymbol{x} ; \sigma_{f}\right)$ means that $S_{\text {line }}$ are calculated by Gaussian $G(x)$ with standard deviation $\sigma_{f}$ on coordinate $\boldsymbol{x}$.

Our method set parameter as $s=1.5, \sigma_{0}=1.5, i=1,2,3, \alpha=0.25$, and $\gamma_{\psi}=\gamma_{\omega}=0.5$ for enhancement hepatic vessels which had over $3[\mathrm{~mm}]$ diameter. 


\subsection{Identification of hepatic vessels}

The value of each voxel in enhanced images in Section 2.2.2 shows the probability of the existence of hepatic vessel regions in CT images. The gray-level thresholding method was applied to identify the hepatic vessel regions from enhanced images. A connected-component labeling and deleting was used to refine the hepatic vessel extraction result by deleting the isolated 3 -D regions with a small volume (litter than 100 voxels).

\section{EXPERIMENTS}

The proposed method was applied to two patient cases of Torso CT images. Each patient case was imaged with a common protocol (120 kV/Auto mA) by a multi-slice CT scanner (UltraSpeed of GE Healthcare). Each CT image covered the whole human torso with about 1000 slices, isotopic spatial resolution of about $0.6 \mathrm{~mm}$ and density (CT number) resolution of 12 bits. The contrast-enhanced CT images for each patient were also prepared as the gold standard for evaluation of hepatic vessels extraction results. A radiologist indicated the truth of the hepatic vessels from the CT images and extraction results by referring the contrast-enhanced CT images slice-by-slice. The result images of each processing step in a patient CT case were shown in Figure 2. The visual evaluation result using contrast enhanced CT images was shown in Figure 3. A hepatic vessels extraction result was shown in Figure 4.

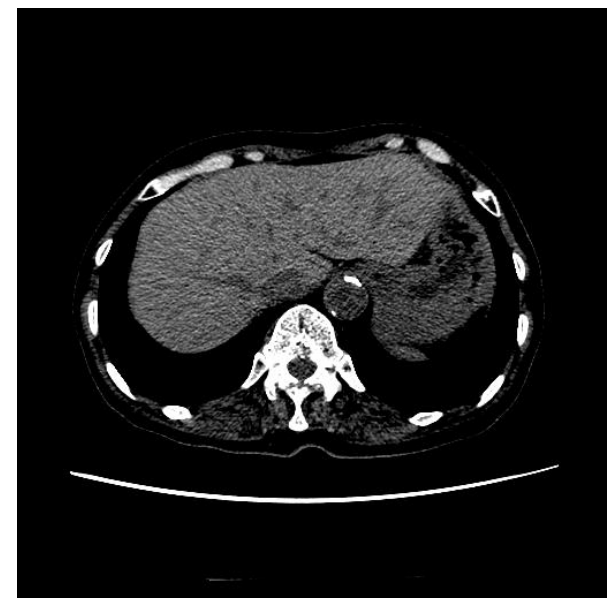

(a)

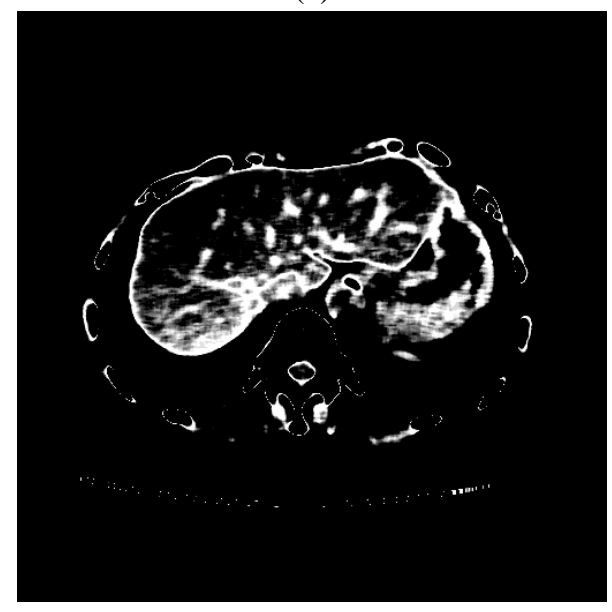

(c)

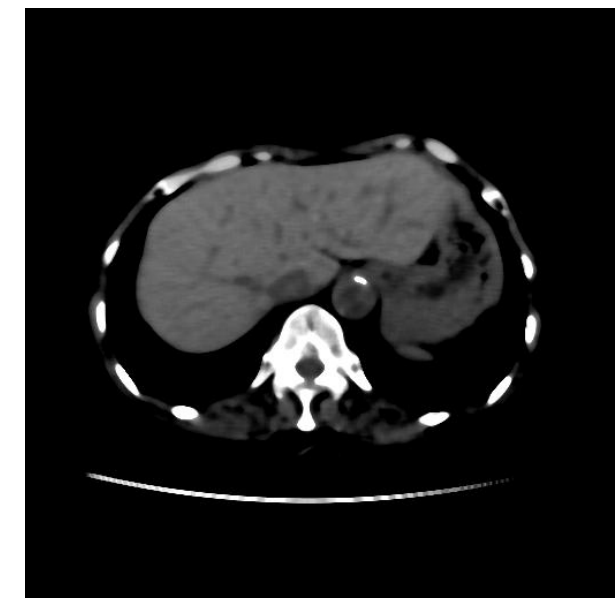

(b)

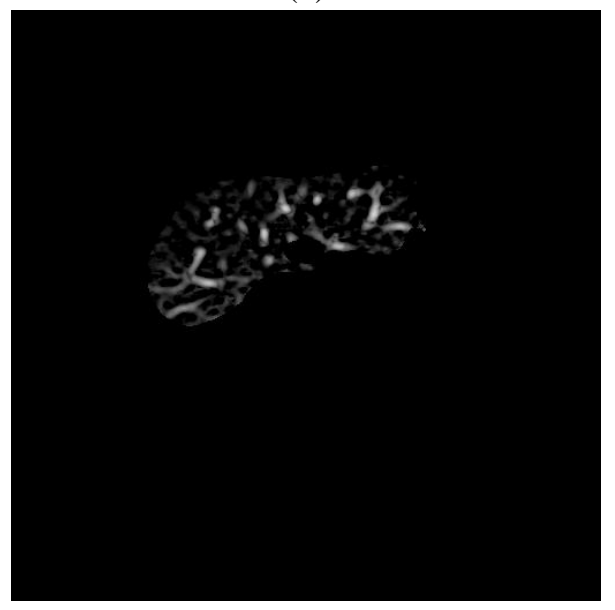

(d)

Figure 2. (a) Original image ( 1 axial slice). (b) After applying median filter (filter size is $7 \times 7 \times 7$ ) to (a). (c) After applying gray-scale windowing function to (b). (d) After applying line components enhancement filtering using eigenvalues of Hessian to (c). 

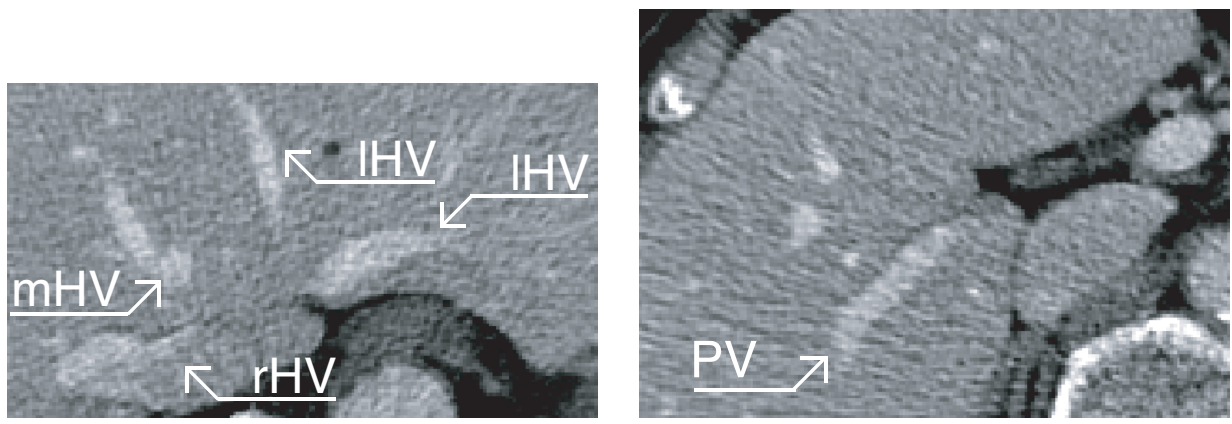

(a)
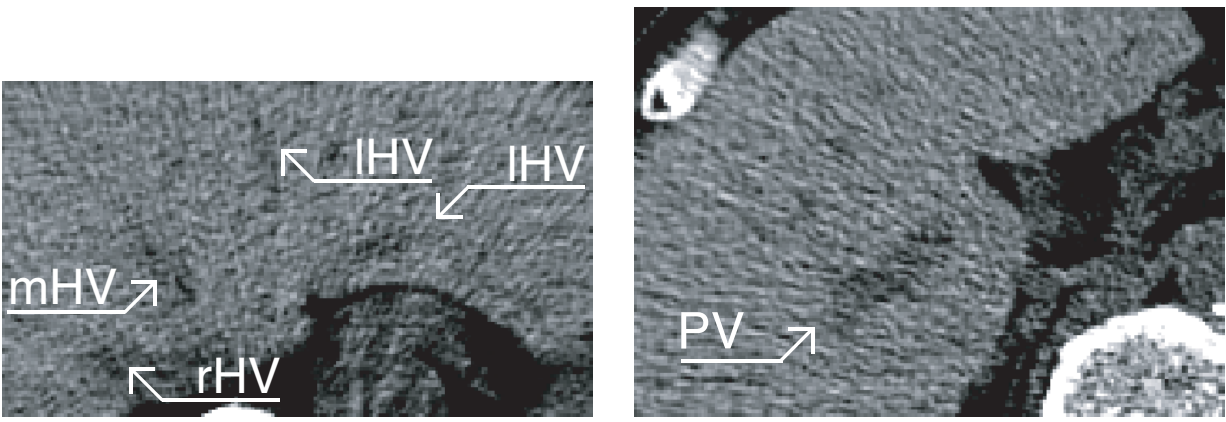

(b)
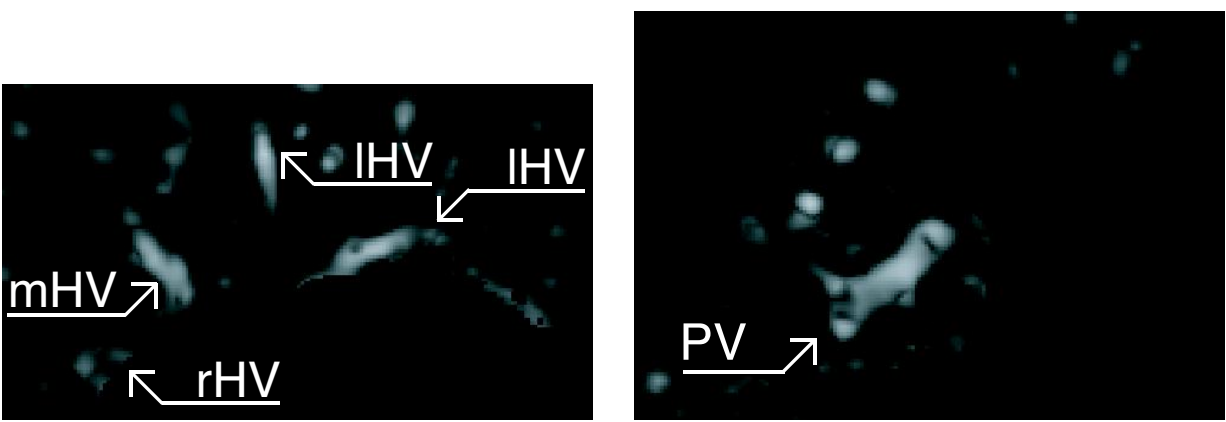

(c)

Figure 3. Comparing the results of liver vessel enhancement. (a) Magnified slices of conventional contrast-enhanced CT images, (b) magnified slices of conventional non-contrast CT images, and (c) magnified slices of virtual contrast-enhanced CT images. (a), (b) and (c) are aligned from left to right with ascending order of slice number of images. Virtual contrast-enhanced means the technique of vessel enhancement proposed in this paper. $\mathrm{rHV}$ (right hepatic vein), mHV(middle hepatic vein), $1 \mathrm{HV}$ (left hepatic vein), and PV(portal vein) in the images indicate the corresponding vessel, respectively.

\section{RESULTS AND DISCUSSION}

Figure 2 shows that transition of a slice of volumes after filtering. Figure 2 (a) is an original image (1 axial slice), Figure 2 (b) is an image after applying median filter (filter size is $7 \times 7 \times 7$ ) to Figure 2 (a), Figure 2 (c) is an image after applying gray-scale windowing function to Figure 2 (b), and Figure 2 (d) is an image after applying line components enhancement filtering using eigenvalues of Hessian to Figure 2 (c) and masking non-liver area.

Hepatic vessels regions can not observed clearly on original CT images as shown in Figure 2 (a). However, Figure 2 (c) showed that our method enhanced the hepatic vessels regions based on the difference of CT numbers between vessel and other tissues on CT images and selected the hepatic vessels correctly as shown in figure 2 (d). 


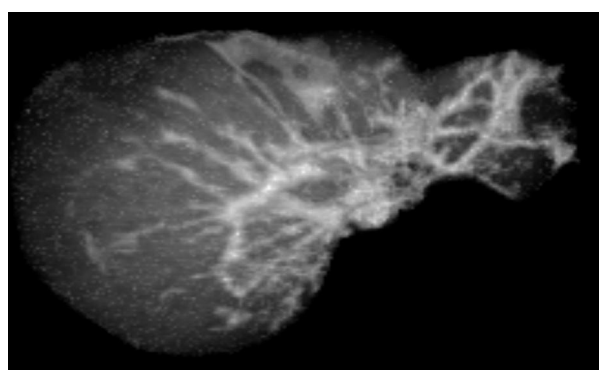

(a)
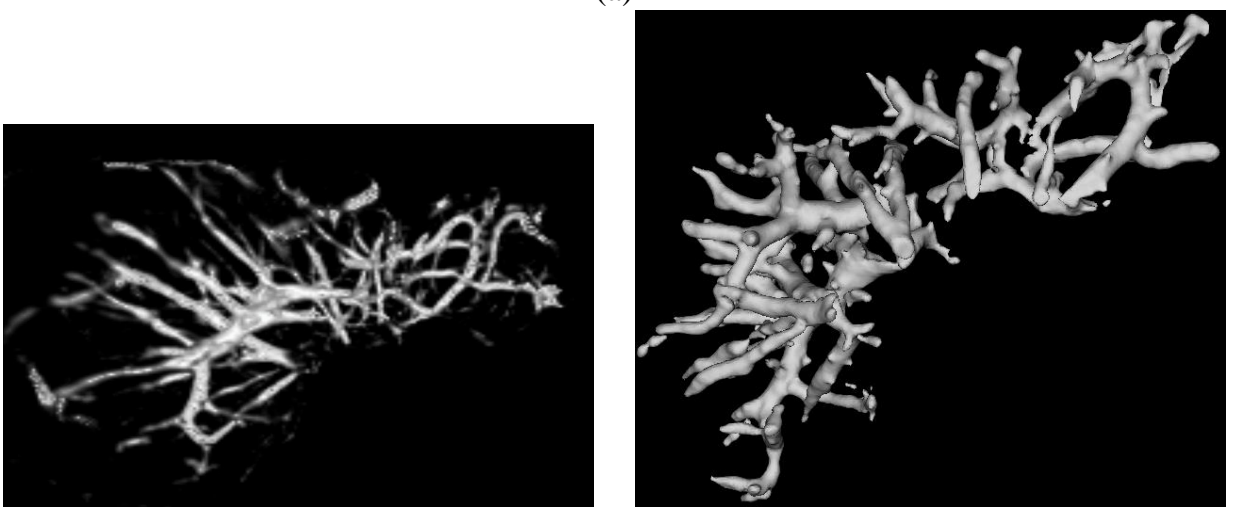

(b)

Figure 4. Volume rendering of liver and hepatic vessel before(a)/after(b) filtering using median filter $(7 \times 7 \times 7)$. (a) Volume rendering of original images. (b) Left: volume rendering. Right: surface rendering of images after removing small volumes. Marching cubes ${ }^{16}$ is used as surface rendering algorithm.

\subsection{Transform of images before/after processing}

It is difficult to distinguish hepatic vessels from original images by gray-scale values visually, because the difference between CT numbers of hepatic vessels and other region of liver is very small. That means the hepatic vessels extraction from non-contrast CT images is also a difficult problem. Using the CT number distribution estimation of vessel regions and line filter based on Hessian matrix, our method showed the potential of the analysis of hepatic vessel tree structures in non-contrast CT images.

\subsection{Enhancement of hepatic vessels}

Figure 3 (a) shows hepatic vessels on contrast-enhanced X-ray CT images, Figure 3 (b) shows hepatic vessels on noncontrast X-ray CT images, and Figure 3 (c) shows result of hepatic vessel enhancement from Figure 3 (b) by the method proposed above, respectively. Figure 3 (a) to (c) are magnified images of a slice which includes parts of hepatic veins (lHV: left hepatic vein, mHV: middle hepatic vein, rHV: right hepatic vein) and a slice which includes parts of portal veins (PV). We compared and evaluated Figure 3 (c). A radiologist notes that areas of noise are recognized as hepatic vessels in Figure 3 (c).

Some of vessel region were lost in the final extraction result. This was due to the misconnection of the hepatic vessels trees after line filtering and some of the vessles regions were removed as the false positives. However, we found that our method preserved the main parts of the hepatic vessel tree on CT images as shown in Figure 4.

In the current research stage, only density (CT number) feature was used as vessel identification. The preliminary results showed that the main part of structures of hepatic vessel tree was recognized [Figure 4 (a) and (b)]. The accuracy of vessel identification will be proved by adjusting the parameters of the line filters and histogram transformation processing and introducing the anatomical knowledge such as shape and topological relations of hepatic vessel tree. 


\section{CONCLUSION}

We proposed a fully-automated method to extract hepatic vessels including portal vein and hepatic vein in non-contrast CT images. The hepatic vessels in liver region were enhanced using a Gaussian-based density transformation and identified using a Hessian-based line filtering. This method was applied to two non-contrast torso CT images. The accuracy evaluations of the vessel extraction were based on visual evaluations by a radiologist specialized in liver diagnosis. The contrast-enhanced CT images of the same patient were used as the ground truth for hepatic vessels evaluation.

We observed that even the density difference between hepatic vessels and other liver regions are very small in noncontrast CT cases; the proposed method can enhance and identify most of the vessels regions as well as human observer can. However, some false-positive regions were occurred in the vessel identification process due to the image noisy. Although we can adjust the parameters of the line filter to reduce the most of the false-positive regions and reserve the principle vessels in liver region, the tips of the vessels were also removed from the vessel extraction results.

We confirmed that the performance of our automated hepatic vessels identification algorithm is comparable to that of human observers. This result has the potential to extract liver lobe and the liver structures (useful for cirrhosis diagnosis) in non-contrast CT images.

\section{ACKNOWLEDGMENTS}

This research is partly supported by Grand-in-Aid for Scientific Research from the Ministry of Education, Culture, Sports, Science, and Technology(MEXT), Japanese Government since 2003.

\section{REFERENCES}

1. D. Selle, B. Preim, A. Schenk, and H.-O. Peitgen, "Analysis of vasculature for liver surgical planning," IEEE Transactions on Medical Imaging 21, pp. 1344-1357, 2002.

2. B. B. Frericks, F. C. Caldarone, B. Nashan, D. H. Savellano, G. Stamm, T. D. Kirchhoff, H.-O. Shin, A. Schenk, D. Selle, W. Spindler, J. Klempnauer, H.-O. Peitgen, and M. Galanski, "3D CT modeling of hepatic vessel architecture and volume calculation in living donated liver transplantation," European Radiology 14, pp. 326-333, 2004.

3. H. Kobatake, A. Shimizu, X. Hu, L.-L. Huang, Y. Hagihara, and S. Nawano, "Simultaneous segmentation of multiple organs in multi-dimensional medical images," in Symposium on Future CAD, Proc. of The First International Symposium on Intelligent Assistance in Diagnosis of Multi-Dimensional Medical Images, pp. I-11-28, 2005.

4. S. Tamura, Y. Sato, Y. Nakajima, T. Johkoh, N. Sugano, and M. Hori, "Computational modeling of organ structures," in Symposium on Future CAD, Proc. of The First International Symposium on Intelligent Assistance in Diagnosis of Multi-Dimensional Medical Images, pp. I-19-24, 2005.

5. Y. Sato, T. Okada, M. Nakamoto, Y. W. Chen, M. Hori, N. Sugano, and S. Tamura, "Computational modeling of anatomical structures," in Symposium on Future CAD, Proc. of The Second International Symposium on Intelligent Assistance in Diagnosis of Multi-Dimensional Medical Images, pp. 42-49, 2006.

6. A. Shimizu, R. Ohno, T. Ikegami, H. Kobatake, S. Nawano, and D. Smutek, "Simultaneous extraction of multiple organs from abdominal CT," in Symposium on Future CAD, Proc. of The Second International Symposium on Intelligent Assistance in Diagnosis of Multi-Dimensional Medical Images, pp. 42-49, 2006.

7. X. Zhang, M. Kanematsu, H. Fujita, T. Hara, and H. Hoshi, "Computerized classification of liver disease in MRI using artificial neural network," Proc. SPIE 4322, pp. 1735-1742, 2001.

8. X. Zhou, T. Hayashi, T. Hara, H. Fujita, R. Yokoyama, T. Kiryu, and H. Hoshi, "Automatic recognition of lung lobes and fissures from multislice CT images," in Medical Imaging, Proc. SPIE 5370, pp. 1629-1633, 2004.

9. X. Zhou, T. Kitagawa, T. Hara, H. Fujita, R. Yokoyama, H. Kondo, M. Kanematsu, and H. Hoshi, "Constructing a probabilistic model for automated liver region segmentation using non-contrast X-ray torso CT images," in Proc. of 9th MICCAI, MICCAI 1, pp. 856-863, 2006.

10. Y. Sato, S. Nakajima, N. Shiraga, H. Atsumi, S. Yoshida, T. Koller, G. Gerig, and R. Kikinis, "Three-dimensional multi-scale line filter for segmentation and visualization of curvilinear structures in medical images," Medical Image Analysis 2, pp. 143-168, 1998.

11. Y. Sato, C.-F. Westin, A. Bhalerao, S. Nakajima, N. Shiraga, S. Tamura, and R. Kikins, "Tissue classification based on 3D local intensity for volume rendering," IEEE Transactions on Visualization and Computer Graphics 6, pp. 160$170,2000$. 
12. W. Tong, C. Tang, P. Mordohai, and G. Medioni, "First order augmentation to tensor voting for boundary inference and multiscale analysis in 3D," IEEE Transactions on pattern analysis and machine intelligence 26, pp. 594-611, 2004.

13. Q. Li, S. Sone, and K. Doi, "Selective enhancement filters for nodules, vessels, and airway walls in two- and threedimensional ct scans," Medical Physics 30, pp. 2040-2051, 2003.

14. B. E. Chapman and D. L. Parker, "3D multi-scale vessel enhancement filtering based on curvature measurements: application to time-of-flight MRA," Medical Image Analysis 9, pp. 191-208, 2005.

15. R. Deriche, "Recursively implementing the gaussian and its derivatives," INRIA Research Report 1893, 1993.

16. W. Lorensen and H. Cline, "Marching cubes: A high resolution 3D surface construction algorithm," Computer Graphics (SIGGRAPH 87) 21, pp. 163-169, 1987. 